\title{
Astrocytes shed extracellular vesicles that contain fibroblast growth factor-2 and vascular endothelial growth factor
}

\author{
PATRIZIA PROIA $^{1 *}$, GABRIELLA SCHIERA ${ }^{2 *}$, MARCO MINEO $^{2}$, ANTONIA MARIA RITA INGRASSIA $^{2}$, \\ GRAZIELLA SANTORO $^{2}$, GIOVANNI SAVETTIERI ${ }^{1}$ and ITALIA DI LIEGRO ${ }^{2}$ \\ ${ }^{1}$ Dipartimento Universitario di Neuroscienze Cliniche and ${ }^{2}$ Dipartimento di Scienze Biochimiche, \\ Università degli Studi di Palermo, Palermo, Italy
}

Received August 7, 2007; Accepted September 14, 2007

\begin{abstract}
We previously set a three-cell-type coculture system in which neurons and astrocytes synergistically induce brain capillary endothelial cells to form a monolayer with permeability properties resembling those of the physiological blood-brain barrier. Moreover, we recently found that neurons produce fibroblast growth factor-2 and vascular endothelial growth factor and secrete them at least in part by shedding extracellular vesicles. In this study, on the basis of immunofluorescence, scanner electron microscopy and Western blot analyses, we concluded that also astrocytes in culture shed extracellular vesicles that contain the same angiogenic factors, as well as $\beta 1$-integrin, a membrane protein that is considered a marker of shedding. Vesicles released by astrocytes are smaller than the ones produced by neurons and have an average size of 150-500 nm.
\end{abstract}

\section{Introduction}

We previously found that neurons and astrocytes cooperate in controlling occludin expression and permeability in brain capillary endothelial cells (BCECs), in a three-cell-type in vitro model of the blood-brain barrier (BBB) (1-3). Since in this culture system physical contacts among the different cell types are not allowed, it is likely that neurons and astrocytes affect endothelial cells by releasing soluble factors. In support of this hypothesis it should be noted that other authors have reported that, in vivo, astrocytes not only form direct contacts with BCECs through astrocyte feet but also release soluble factors, such as basic fibroblast growth factor (bFGF or FGF-2) and vascular endothelial growth factor

Correspondence to: Dr Italia Di Liegro, Dipartimento di Scienze Biochimiche, Università degli Studi di Palermo, Via del Vespro 129, I-90127 Palermo, Italy

E-mail: diliegro@unipa.it

${ }^{*}$ Contributed equally

Key words: astrocytes, extracellular vesicle shedding, vascular endothelial growth factor, fibroblast growth factor-2
(VEGF) (4), which find receptors on BCECs. FGF-2 is a protein that lacks a standard signal sequence and cannot be sorted to the endoplasmic reticulum (5). It has been reported to be secreted by tumor cells through an unusual way, which involves shedding of extracellular vesicles from the plasma membrane (5). FGF-2 is a potent inducer of blood vessel formation (angiogenesis), with a fundamental role in the development and differentiation of various tissues $(6,7)$, including the nervous system (7). In addition to a main polypeptide of $18 \mathrm{kDa}$, other FGF-2 isoforms have been described, ranging in size from 22 to $34 \mathrm{kDa}$ (7).

VEGF, another factor with well-known actions on endothelial cells, has also been reported to be associated with extracellular vesicles released by tumor cells $(5,8)$. VEGF exists as a homodimer of isoforms of different sizes (i.e. 121, $145,165,189,206)$, derived from alternative splicing of the same primary transcript (9). In addition to these smaller forms, a larger isoform, with an N-terminal extension of 200 amino acids and an apparent mass of $45 \mathrm{kDa}$ has been described in the human and the mouse $(10,11)$.

As it has been recently found that some glial tumor cells (oligodendroglioma cells) (12) as well as primary neurons in culture (13) are able to release extracellular vesicles, we investigated the possibility that astrocytes can do the same. We found that indeed astrocytes produce extracellular structures that contain FGF-2 and VEGF, together with B1integrin, a membrane protein reported by other authors (see, for example: 14) to be a component of a heterogeneous population of large extracellular vesicles, ranging in size from 100-1000 nm called microvesicles (MVs).

\section{Materials and methods}

Animals. Procedures involving animals were conducted according to the European Community Council Directive 86/609, OJL 358 1, December 12, 1987. Winstar rats (Stefano Morini, San Paolo d'Enza, Italy) were housed in the institutional animal care facility of the Department of 'Biologia Cellulare e Sviluppo', University of Palermo, Palermo, Italy, under the direction of a licensed veterinarian who approved the protocols.

Cell cultures and vesicle purification from conditioned medium. Astrocytes were purified from brain cortices of 2- 
day-old rats, as described by Cole and de Vellis (15), exploiting the differences in adhesion of the various cells and cultured on fibronectin, in DME/Ham's F12 (2:1), supplemented with $10 \%$ heat-inactivated fetal calf serum, until half-confluence (1). Astrocytes were then progressively adapted to Maat medium (MM) (16). After $24 \mathrm{~h}$ of incubation, conditioned medium was collected and centrifuged to obtain vesicles, as previously described $(13,17)$. Briefly, media were centrifuged at $2000 \mathrm{x} \mathrm{g}$ for $10 \mathrm{~min}$ and $4000 \mathrm{x} \mathrm{g}$ for $15 \mathrm{~min}$. The last supernatant was finally centrifuged at 105,000 x g for $90 \mathrm{~min}$. Pelleted vesicles were re-suspended in PBS. In certain experiments, BSA-containing medium was withdrawn and substituted with BSA-free fresh medium, and after $3 \mathrm{~h}$ of incubation, the medium was collected and vesicles were prepared as described above. Finally, after 10 days of culture, astrocytes were lysed to obtain total cell proteins.

Western blot analyses. Astrocytes were homogenized in homogenization buffer $(0.32 \mathrm{M}$ sucrose; $50 \mathrm{mM}$ sodium phosphate buffer, $\mathrm{pH} 6.5 ; 50 \mathrm{mM} \mathrm{KCl} ; 0.5 \mathrm{mM}$ spermine; $0.15 \mathrm{mM}$ spermidine; $2 \mathrm{mM}$ EDTA; and $0.15 \mathrm{mM}$ EGTA), containing the protease inhibitors aprotinin $(2 \mu \mathrm{g} / \mathrm{ml})$, antipain $(2 \mu \mathrm{g} / \mathrm{ml})$, leupeptin $(2 \mu \mathrm{g} / \mathrm{ml})$, pepstatin A $(2 \mu \mathrm{g} /$ $\mathrm{ml})$, benzamidine $(1.0 \mathrm{mM})$, and phenylmethylsulfonyl fluoride (1.0 mM) (all from Sigma-Aldrich, MO, USA).

Proteins $(20 \mu \mathrm{g}$ of total cell extracts) were separated by electrophoresis on denaturing $15 \%$ polyacrylamide slab gels (SDS-PAGE), immunoblotted on PVDF membrane (Immobilon P, Millipore, MA, USA) and immunostained with rabbit polyclonal anti-human VEGF antibodies or rabbit polyclonal anti-FGF-2 (both from Santa Cruz, CA, USA).

Scanner electron microscopy (SEM). Astrocytes were cultured on coverslips for 10 days in MM. Cells were rinsed with PBS at $37^{\circ} \mathrm{C}$ and fixed with $4 \%$ glutaraldehyde in $\mathrm{MM}$ for $10 \mathrm{~min}$. After rinsing with MM, cells were dehydrated by a series of washes in acetone/water at increasing percentages of acetone $(15,30,50,75$ and $100 \%$; 3 min each). Coverslips were finally dried in air, coated with gold and examined with a Philips 505 scanner electron microscope.

Immunofluorescence. Astrocytes were cultured on coverslips for 10 days, fixed with $96 \%$ ethanol on ice for $10 \mathrm{~min}$ and permeabilized for 5 min with $0.1 \%$ Triton X-100 in PBS. Cells were finally incubated with: i) rabbit polyclonal antihuman VEGF antibodies, ii) rabbit polyclonal anti-FGF-2 antibodies, iii) goat anti-integrin $\beta 1$ (Santa Cruz, CA, USA) antibodies; or iv) polyclonal rabbit anti-glial fibrillary acidic protein (GFAP) antibodies (Sigma-Aldrich, MO, USA). The secondary antibodies were anti-rabbit or anti-goat IgGs, conjugated to rodhamine or to fluoresceine (Promega Corp., WI, USA). Astrocytes were observed with an Olympus Fluoview FV300 confocal microscope, equipped with two laser multipliers. In some cases, cells prepared as described were finally stained for DNA by treating with Vectashield mounting medium for fluorescence containing 4',6-diamino2-phenylindole (Vector Laboratories, Youngstown, OH, USA), and observed with an Olympus BX-50 microscope (Olympus Italia S.r.l., Segrate, Italy) equipped with a Vario Cam B/W camera (Nikon Instruments S.p.A., Calenzano, Italy).
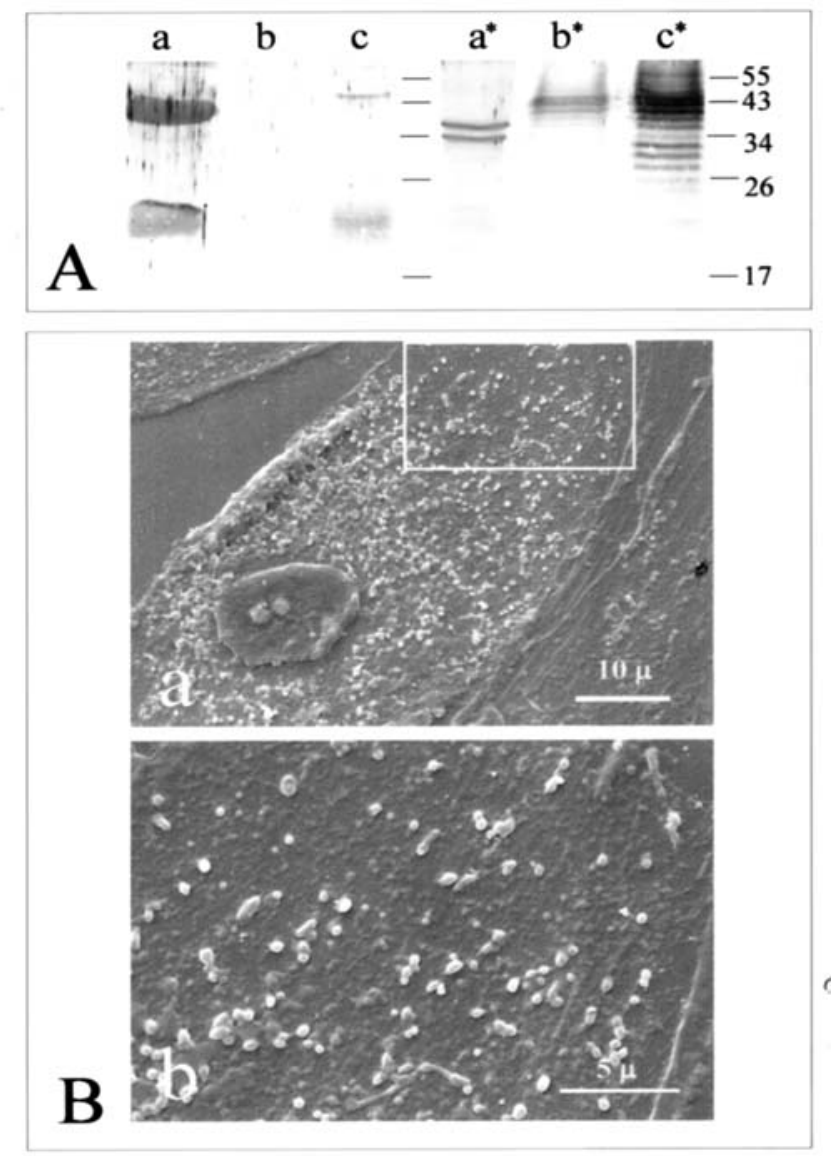

Figure 1. Astrocytes, cultured for 10 days in serum-free Maat medium, released extracellular vesicles. (A) Western blot analysis of total cell lysates (lanes $a$ and $a^{*}$ ) and vesicles (lanes $b, c, b^{*}$ and $c^{*}$ ) prepared from astrocytes cultured only in BSA-containing Maat medium (lanes $b$ and $b^{*}$ ) or from astrocytes cultured in BSA-containing Maat medium until the last $3 \mathrm{~h}$ before purifying the vesicles (lanes $\mathrm{c}$ and $\mathrm{c}^{*}$ ). Proteins were immunostained with either rabbit polyclonal anti-human VEGF antibodies (lanes a-c) or rabbit polyclonal anti-FGF-2 (lanes $\left.a^{*}-c^{*}\right)$. (B) Analysis by scanner microscopy of astrocyte cultures. Cells produced and released extracellular vesicular structures. A detailed section (the white box in a) was observed at a higher magnification (b).

\section{Results}

Tumor cells of glial origin were recently shown to release extracellular vesicles that contain the FAS ligand and induce apoptosis in rat cortical neurons in culture (12). We also found that neurons themselves can shed large extracellular vesicles that contain angiogenic factors such as FGF-2 and VEGF (13). As neurons and astrocytes were found to regulate, even in the absence of direct cell-to-cell contacts, the ability of endothelial cells in culture to form a monolayer with BBB properties, we attempted to ascertain whether astrocytes are also able to release angiogenic factors by shedding extracellular vesicles.

When we centrifuged the medium in which astrocytes had been cultured, we indeed obtained a 'vesicular' fraction that contained two proteins ( 45 and $22 \mathrm{kDa}$, respectively) immunostained by anti-VEGF (Fig. 1A, lane c). These proteins seemed to be present in higher amounts in astrocyte total lysates. In the vesicular fraction, some proteins were also present that could be stained by anti-FGF-2 antibodies 


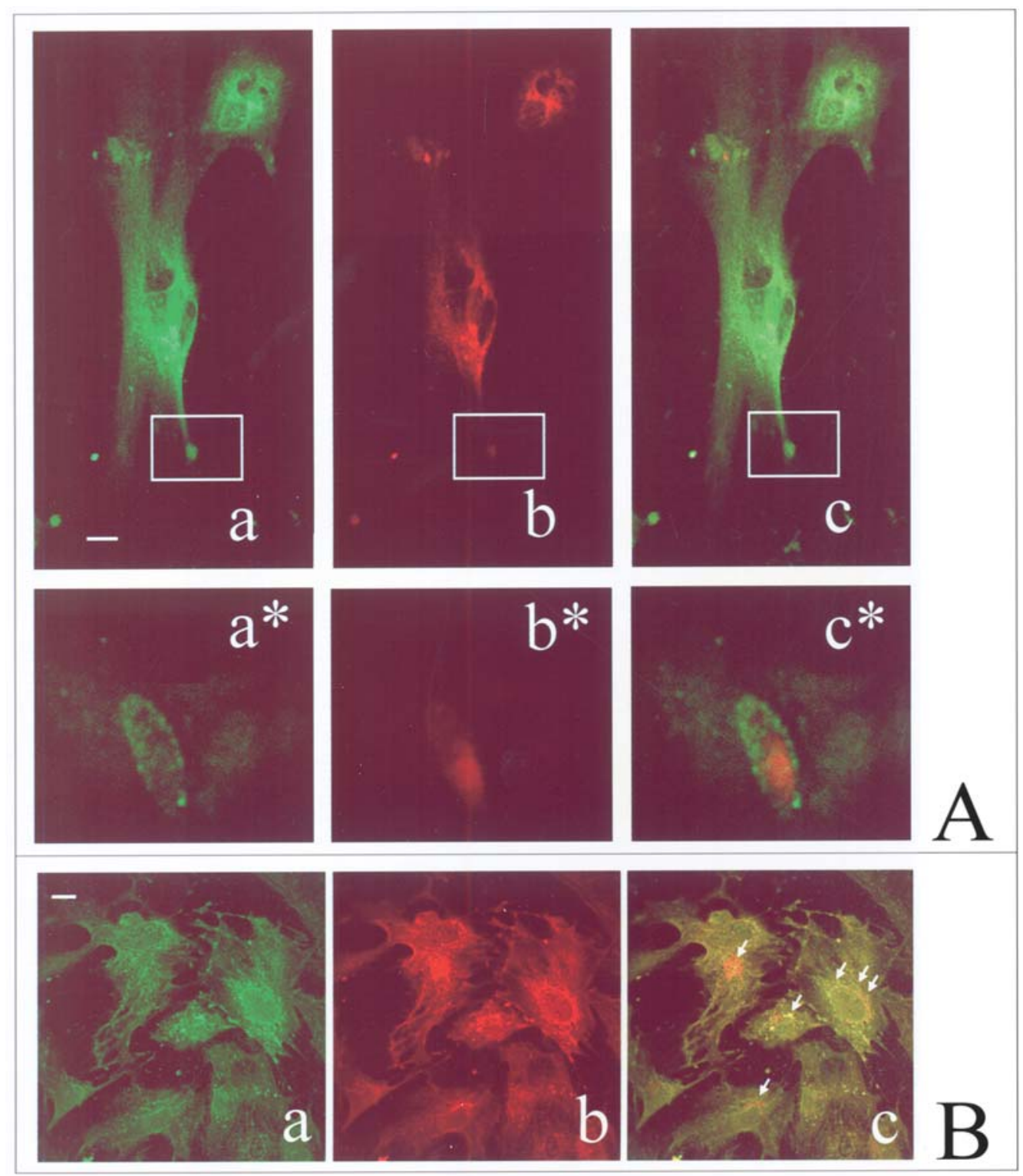

Figure 2. Double immunofluorescence of VEGF and either GFAP (A) or B1-integrin (B). (A) Localization of GFAP (green fluorescence, a and a*) and VEGF (red fluorescence, $\mathrm{b}$ and $\mathrm{b}^{*}$ ) in astrocytes cultured for 10 days in Maat medium. The overlay of red and green fluorescence is shown in $\mathrm{c}$ and $\mathrm{c}^{*}$. Enlarged views of the fields boxed in figures $a-c$ are shown in $\mathrm{a}^{*}-\mathrm{c}^{*}$. (B) Colocalization of $\beta 1$-integrin (a, green) and VEGF (b, red) immunoreactivity in astrocytes cultured for 10 days in Maat medium. Overlay of VEGF and B1-integrin fluorescence is shown in c. Cells were observed with an Olympus Fluoview FV300 confocal microscope, equipped with two laser multipliers. Bar, $10 \mu \mathrm{m}$.

(Fig. 1A, lanes $\mathrm{b}^{*}$ and $\mathrm{c}^{*}$ ). In particular, a series of bands, ranging in size from $21-43 \mathrm{kDa}$, were evident. To note, in the case of FGF-2, some of the immunostained proteins were barely visible in the total astrocyte lysates (Fig. 1A, lane a*), where other proteins were instead stained at a higher level: two main bands of 34 and $36 \mathrm{kDa}$, respectively, and a group of three faint bands with apparent masses of $\sim 18-22 \mathrm{kDa}$. In addition, in the vesicle fractions purified from complete Maat medium, which contained albumin, it was difficult to obtain a clear profile of reacting proteins by Western blot analysis (Fig. 1A, lanes $\mathrm{b}$ and $\mathrm{b}^{*}$ ). On the other hand, when BSAcontaining medium was withdrawn and substituted with BSA-free fresh medium $3 \mathrm{~h}$ before collecting vesicles, Western blot analysis gave clearer results (Fig. 1A, lanes c and $\mathrm{c}^{*}$ ). Staining of the membranes by Ponceau Red (not shown) revealed that the 'vesicular' fraction also contained albumin, possibly absorbed to lipid components of vesicles; the presence of albumin caused a relative dilution of the other proteins present in the vesicular fraction. The fact that astrocytes produce extracellular structures was also demonstrated by scanner electron microscopy (Fig. 1B), where vesicles of $\sim 150-500 \mathrm{~nm}$ were clearly visible. On average, the vesicles produced are smaller than the ones released by neurons (400-2000 $\mathrm{nm})(13)$, but are still in the size class of MVs. However, an enlarged view of the vesicles (Fig. 1 $\mathrm{Bb}$ ) showed that some very minute structures were also present in the vesicular population. Thus, it is possible that exosomes were also produced.

Once we proved that astrocytes released extracellular vesicles that seemed to contain VEGF as well as FGF-2, we investigated for localization of membrane domains involved in shedding by confocal microscopy. Fig. 2 shows results of 

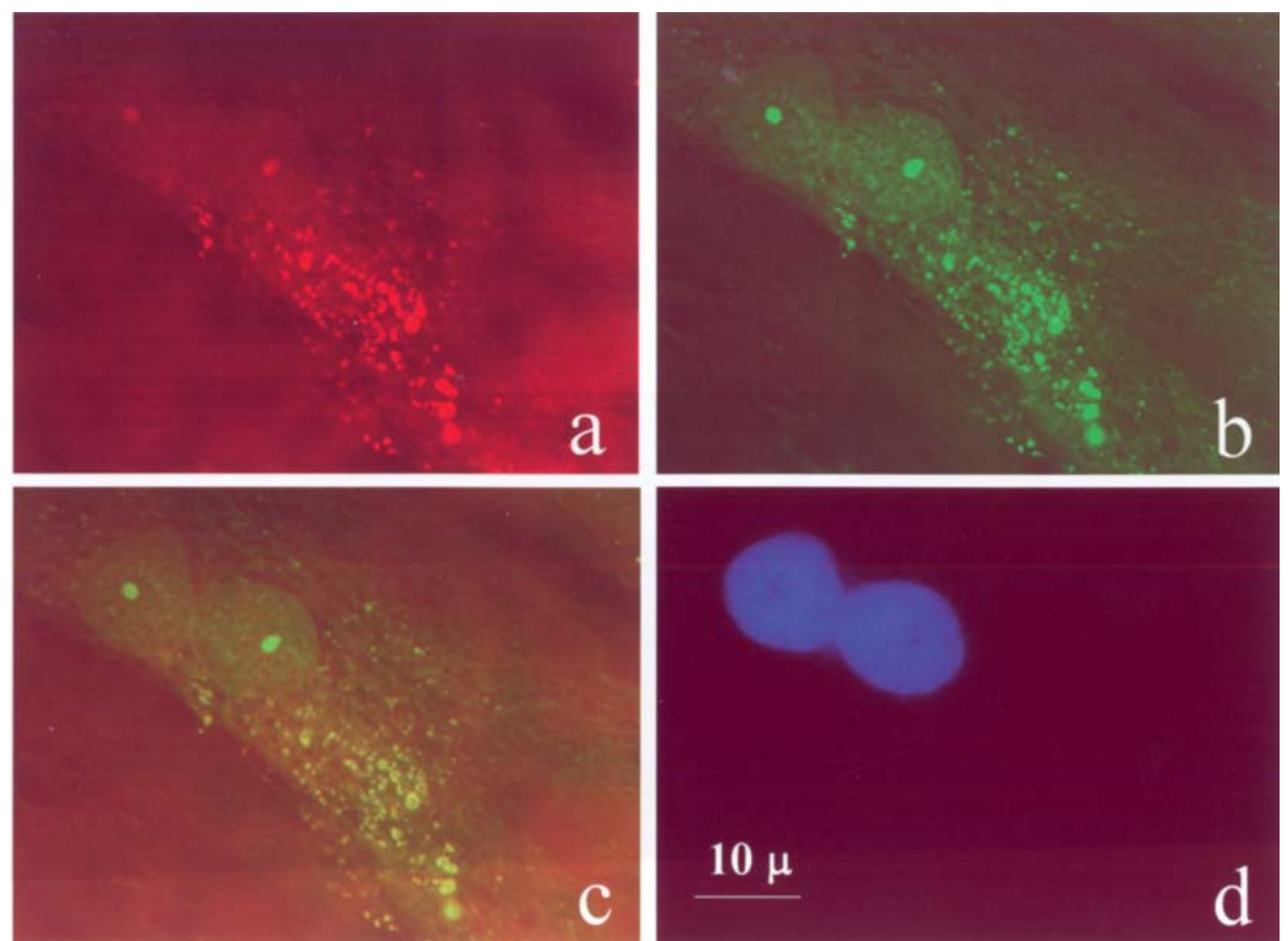

Figure 3. Colocalization of FGF-2 with ß1-integrin in extracellular vesicles. Double immunofluorescence was used to colocalize FGF-2 (a, red) and ß1integrin (b, green) immunoreactivity in astrocytes cultured for 10 days in Maat medium. Overlay of FGF-2 and B1-integrin fluorescence is shown in c. Cells were finally stained for DNA (d, blue), by treating with Vectashield mounting medium for fluorescence, containing 4',6-diamino-2-phenylindole (DAPI), and were observed with an Olympus BX-50 microscope equipped with a Vario Cam B/W camera.

analyses in which astrocytes were stained with anti-VEGF antibodies and with antibodies directed against either the astrocyte-specific intermediate filament protein GFAP (A) or the membrane protein $B 1$-integrin (B). Although a diffused intracellular red staining was observed (Fig. 2A, lane b and Fig. 2Bb), red spots were in particular visible at the level of small round structures, some of which had an astrocyte footlike appearance (Fig. 2Aa*-c*), while others could be interpreted as extracellular vesicles stained for both $\beta 1$ integrin and VEGF (Fig. 2Bc, yellow fluorescence). In most cases, the extracellular membranous structures formed a crown around a central position of the cells, probably over the putative position of the nucleus (Fig. 2B, structures indicated by arrows).

Other cells were immunostained for FGF-2 (Fig. 3). Extracellular membranous structures, which contained both ß1-integrin and FGF-2, were clearly visible. Since we recently found that extracellular vesicles produced by neurons are not stained by the DNA-specific dye DAPI, and that this property seems to distinguish them from apoptotic bodies (13), we stained astrocytes with DAPI as well. As shown in Fig. 3d, vesicles were not stained.

\section{Discussion}

We previously demonstrated that neurons and astrocytes control the ability of brain capillary endothelial cells to form in culture a barrier with permeability properties resembling those of the physiological BBB $(1,2,18,19)$. Since in the three-cell-type culture system used physical contacts among the different cell types are not allowed, it is likely that the effects discovered were not based on direct cell-to-cell contacts. Therefore, we investigated the possibility that either neurons and/or astrocytes produce extracellular factors responsible for the observed effects. We looked in particular for the angiogenic proteins FGF-2 and VEGF and investigated the possibility that these factors are released by extracellular vesicle shedding. On the basis of different techniques, this study allows us to conclude that astrocytes shed extracellular vesicles of different sizes, from $\sim 150-500 \mathrm{~nm}$. These dimensions, together with the fact that vesicles contained $\beta 1$-integrin, suggest that they can be classified as MVs. The population of extracellular structures is, however, heterogeneous and we cannot exclude, at the moment, that exosomes can be also produced. We also report that the extracellular vesicles shed by astrocytes, similar to those shed by neurons (13), contained the angiogenic factors VEGF and FGF-2. In more detail, the Western blot analyses indicated that vesicles contained two main VEGF isoforms ( $\sim 45$ and $22 \mathrm{kDa}$, respectively). The situation concerning FGF-2 was more complex. In this case, we observed a series of bands, ranging in size from 21 to $43 \mathrm{kDa}$, some of which were barely visible in the total astrocyte lysates (Fig. 1A, lane a*). On the other hand, in the total cell lysates, two main bands of 34 and $36 \mathrm{kDa}$, respectively, and a group of three faint bands with apparent masses of $\sim 18-22 \mathrm{kDa}$ were intensively stained. Thus, for particular reasons, only certain FGF-2 isoforms were sorted to vesicles, while the others remained in the cell, where perhaps they have different functions that, according to previous reports, might involve nuclear 
localization (7). To note, the pattern of VEGF- and FGF-2like bands, observed in astrocytes, only in part overlap that observed in neurons (13), confirming the possibility that different isoforms might have different functions.

We also report that for vesicles purified from complete Maat medium, somehow albumin hampered the observation of less represented proteins in the Western blot analysis (Fig. 1A, lanes b and $\mathrm{b}^{*}$ ). When, indeed, cells were cultured with BSA-free fresh medium for $3 \mathrm{~h}$ before collecting vesicles, Western blot analyses gave clearer results (Fig. 1A, lanes $\mathrm{c}$ and $\mathrm{c}^{*}$ ).

This study showed that astrocytes, like neurons (13), use a novel mechanism to release factors that contribute to brain cell differentiation and function. This mechanism, first discovered in tumor cells, has been recently suggested, on the basis of many lines of evidence, to constitute a general route of cell to cell communications (20-22).

Notably, in the case of astrocytes, it seems that the release of extracellular vesicles involves, at least in part, cell domains that could be interpreted as astrocytic feet. This finding might shed new light on the functions of these unique astrocyte structures. We are now investigating what occurs when vesicles produced by neurons or astrocytes are added to other brain cell cultures.

\section{Acknowledgements}

We wish to thank Dr F. Carfi Pavia and Mr. S Agnello for their helpful assistance with scanner electron microscopy and confocal microscopy, respectively. This work was supported by Serono S.p.A., and by the University of Palermo (fondi di Ateneo, Università degli Studi di Palermo). P. Proia was supported by a Ph.D fellowship of the Università degli Studi di Palermo, Palermo, Italy. G. Schiera was supported by a postdoctoral fellowship by Università degli Studi di Palermo, Palermo, Italy.

\section{References}

1. Schiera G, Bono E, Raffa MP, Gallo A, Pitarresi GL, Di Liegro I and Savettieri G: Synergistic effects of neurons and astrocytes on the differentiation of brain capillary endothelial cells in culture. J Cell Mol Med 7: 165-170, 2003.

2. Schiera G, Sala S, Gallo A, Raffa MP, Pitarresi GL, Savettieri G and Di Liegro I: Permeability properties of a three-cell type in vitro model of blood-brain barrier. J Cell Mol Med 9: 373-379, 2005.

3. Schiera $\mathrm{G}$ and Proia P: In vitro models of blood-brain barrier formation and functioning. In: Molecular Bases of Neurodegeneration. Di Liegro I and Savettieri G (eds). Research Signpost, Kerala, pp183-197, 2005.

4. Nakagawa T and Schwartz JP: Gene expression patterns in vivo normal adult astrocytes compared with cultured neonatal and normal adult astrocytes. Neurochem Int 45: 203-242, 2004.
5. Taverna S, Ghersi G, Ginestra A, Rigoglioso S, Pecorella S, Alaimo G, Saladino F, Dolo V, Dell'Era P, Pavan A, Pizzolanti G, Mignatti P, Presta M and Vittorelli ML: Shedding of membrane vesicles mediates fibroblast growth factor-2 release from cells. J Biol Chem 278: 51911-51919, 2003.

6. Basilico C and Moscatelli D: The FGF family of growth factors and oncogenes. Adv Cancer Res 59: 115-165, 1992.

7. Bikfalvi A, Klein S, Pintucci G and Rifkin DB: Biological roles of fibroblast growth factor-2. Endocr Rev 18: 26-45, 1997.

8. Taraboletti G, D'Ascenzo S, Giusti I, Marchetti D, Borsotti P, Millimaggi D, Giavazzi R, Pavan A and Dolo V: Bioavailability of VEGF in tumor-shed vesicles depends on vesicle burst induced by acidic pH. Neoplasia 8: 96-103, 2006.

9. Ferrara N and Davis-Smith T: The biology of vascular endothelial growth factor. Endocr Rev 18: 4-25, 1997.

10. Huez I, Bornes S, Bresson D, Créancier L and Prats H: New vascular endothelial isoform generated by internal ribosomal entry site-driven CUG translation initiation. Mol Endocrinol 15: 2197-2210, 2001

11. Tee MK and Jaffe RB: A precursor form of vascular endothelial growth factor arises by initiation from an upstream in-frame CUG codon. Biochem J 359: 219-226, 2001.

12. D'Agostino S, Salomone M, Di Liegro I and Vittorelli ML: Membrane vesicles shed by oligodendroglioma cells induce neuronal apoptosis. Int J Oncol 29: 1075-1085, 2006.

13. Schiera G, Proia P, Alberti C, Mineo M, Savettieri G and Di Liegro I: Neurons produce FGF2 and VEGF and secrete them at least in part by shedding extracellular vesicles. J Cell Mol Med (In press).

14. Dolo V, Ginestra A, Cassara D, Violini S, Lucania G, Torrisi MR, Nagase H, Canevari S, Pavan A and Vittorelli ML: Selective localization of matrix metalloproteinase 9 , beta1 integrins, and human lymphocyte antigen class I molecules on membrane vesicles shed by 8701-BC breast carcinoma cells. Cancer Res 58: 4468-4474, 1998.

15. Cole R and de Vellis J: Preparation of astrocytes and oligodendrocyte cultures from primary rat glial cultures. In: A Dissection and Tissue Culture Manual of the Nervous System. Alan R Liss Inc, New York, pp121-133, 1989.

16. Cestelli A, Savettieri G, Ferraro D and Vitale F: Formulation of a novel synthetic medium for selectively culturing rat CNS neurons. Dev Brain Res 22: 219-227, 1985.

17. Dolo V, Ginestra A, Ghersi G, Nagase H and Vittorelli ML: Human breast carcinoma cells cultured in the presence of serum shed membrane vesicles rich in gelatinolytic activities. J Submicrosc Cytol Pathol 26: 173-180, 1994.

18. Savettieri G, Di Liegro I, Catania C, Licata L, Pitarresi GL, D'Agostino S, Schiera G, De Caro V, Giandalia G, Giannola LI and Cestelli A: Neurons and ECM regulate occludin localization in brain endothelial cells. Neuroreport 11: 1081-1084, 2000.

19. Cestelli A, Catania C, D'Agostino S, Di Liegro I, Licata L, Schiera G, Pitarresi GL, Savettieri G, De Caro V, Giandalia G and Giannola LI: Functional feature of a novel model of blood brain barrier: studies on permeation of test compounds. J Control Release 76: 139-147, 2001.

20. Vittorelli ML: Shed membrane vesicles and clustering of membrane-bound proteolytic enzymes. Curr Top Dev Biol 54: 411-432, 2003.

21. Pilzer D, Gasser O, Moskovich O, Schifferli JA and Fishelson Z: Emission of membrane vesicles: roles in complement resistance, immunity and cancer. Springer Semin Immunopathol 27: 375-387, 2005.

22. Whiteside TL: Tumour-derived exosomes or microvesicles: another mechanism of tumour escape from the host immune system? Br J Cancer 92: 209-211, 2005. 